\title{
DYNAMIC RESPONSE OF A SIMPLY SUPPORTED VISCOELASTIC BEAM OF A FRACTIONAL DERIVATIVE TYPE TO A MOVING FORCE LOAD
}

\author{
JAN FREUNDLICH \\ Warsaw University of Technology, Faculty of Automotive and Construction Machinery Engineering, Warszawa, Poland \\ e-mail: jfr@simr.pw.edu.pl
}

In the paper, the dynamic response of a simply supported viscoelastic beam of the fractional derivative type to a moving force load is studied. The Bernoulli-Euler beam with the fractional derivative viscoelastic Kelvin-Voigt material model is considered. The RiemannLiouville fractional derivative of the order $0<\alpha \leqslant 1$ is used. The forced-vibration solution of the beam is determined using the mode superposition method. A convolution integral of fractional Green's function and forcing function is used to achieve the beam response. Green's function is formulated by two terms. The first term describes damped vibrations around the drifting equilibrium position, while the second term describes the drift of the equilibrium position. The solution is obtained analytically whereas dynamic responses are calculated numerically. A comparison between the results obtained using the fractional and integer viscoelastic material models is performed. Next, the effects of the order of the fractional derivative and velocity of the moving force on the dynamic response of the beam are studied. In the analysed system, the effect of the term describing the drift of the equilibrium position on the beam deflection is negligible in comparison with the first term and therefore can be omitted. The calculated responses of the beam with the fractional material model are similar to those presented in works of other authors.

Keywords: fractional viscoelasticity, moving loads, beam vibrations, transient dynamic analysis

\section{Introduction}

The problem of predicting the dynamic response of a structure resulting from the passage of moving loads often occurs in engineering analysis. This problem has many applications in engineering analysis, some of these applications are vibrations that occur in bridges and railroad tracks, cranes, machine elements, weapon barrels, rails, rail and vehicle body parts. The studies show that the transversal deflection and stresses due to moving loads are considerably higher than those observed with stationary loads. The dynamic behaviour of a beam subjected to moving loads or moving masses have been studied extensively in connection with the design of railway tracks and bridges as well as machining processes. A broad overview of the analytical and numerical methods deals with these issues is provided in a book by Fryba (1972). A newer survey of these methods can be found e.g. in a book by Bajer and Dyniewicz (2012). The book also presents a comprehensive overview of the numerical methods including the finite element method (FEM) and the space-time element method (STEM). Additionally, both these books contain a broad bibliography devoted to the dynamic analysis of structures subjected to moving loads.

Furthermore, damping effects on structure dynamics are considered in many works devoted to the mentioned above subject (e.g Fryba, 1972). Therefore, selection of an appropriate viscoelastic material model is an essential element in studying the dynamical behaviour of mechanical structures. The selected model should as accurately as possible describe viscoelastic damping 
over a wide interval of frequencies. A large number of engineering materials show a weak frequency dependence of their damping properties within a broad frequency range (Bagley and Torvik, 1983a,b; Caputo and Mainardi, 1971a; Clough and Penzien, 1993). This weak frequency dependence is difficult to describe using classical viscoelastic models, based on integer order rate laws. In the recent years, the fractional derivatives have been frequently utilized to describe dynamic characteristics of viscoelastic materials and damping elements (Podlubny, 1999; Mainardi, 2009; Rossikhin and Shitikova, 2010; Hedrih (Stevanović) 2014; Hedrih (Stevanović) and Machado, 2015). By introducing fractional-order derivatives instead of integer-order derivatives in the constitutive relations, the number of parameters required to accurately describe the dynamic properties can be significantly reduced (see, e.g., Caputo and Mainardi, 1971b; Bagley and Torvik, 1983b; Enelund and Olsson, 1999). A review of publications dedicated to application of fractional calculus in dynamics of solids can be found in the paper by Rossikhin and Shitikova (2010). Moreover, a fractional damping is utilized in dynamical analysis of beams under moving loads (e.g. Abu-Mallouh et al., 2012; Alkhaldi et al., 2013). In these papers, taken into consideration are only loads moving at a constant speed. The solutions presented in these works are obtained with the help of Mittag-Lefler functions and infinite series. In numerous studies in which fractional viscoelastic models are employed, the dynamic transient analysis is limited to the response induced by initial conditions. However, structures subjected to moving loads with a time varying velocity are often encountered in engineering practice. The number of publications considering the dynamic behaviour of a beam with a fractional viscoelstic model under moving loads is rather limited. Numerous research works related to structure vibrations described by fractional models present transient solutions expressed as series expansions (Atanackovic and Stankovic, 2002; Bagley and Torvik, 1986; Caputo, 1974; Hedrih (Stevanović) and Filipovski, 2002; Podlubny, 1999) which are not very useful for practical applications because of their slow convergence rate (Podlubny, 1999; Rossikhin and Shitikova, 2010). Thus, the aim of this work is demonstration of an approach utilizing fractional Green's function in dynamical analysis of a simply-supported beam subjected to a moving force with a constant velocity and constant acceleration. Green's function is evaluated using a closed contour of integration in conjunction with the residue theorem (Caputo and Mainardi, 1971b; Bagley and Torvik, 1983b, 1986; Rossikhin and Shitikova, 1997; Beyer and Kempfle, 1995). The proposed approach is the expansion of methods employing fractional calculus in the modelling of damping properties of dynamic systems.

\section{Problem formulation}

The equation of motion of the examined beam is derived on the assumption of the Bernoulli-Euler theory, neglecting rotary inertia and shear deformation. Furthermore, it is assumed that the beam is homogeneous with constant cross-section along its length, and the neutral axis of the beam bending is one of the principal axes of inertia of the normal cross section of the beam. Beam oscillations are assumed in the $x y$ plane (Fig. 1). Moreover, it is assumed that the internal dissipation of mechanical energy in the beam material is described by a differential equation of the fractional order. Therefore, the stress-strain constitutive relation of the beam material is in the following form (e.g. Bagley and Torvik, 1983a)

$$
\sigma=E \varepsilon(t)+E_{\gamma}^{\prime} D_{t}^{\gamma}[\varepsilon(t)]=E\left(\varepsilon+\mu_{\gamma} \frac{d^{\gamma} \varepsilon(t)}{d t^{\gamma}}\right)
$$

where: $\mu_{\gamma}=E_{\gamma}^{\prime} / E, E$ - Young's modulus of the beam material, $E_{\gamma}^{\prime}$ - damping coefficient, $D_{t}^{\gamma}[\cdot]$ - fractional order differential operator of the $\gamma$-th derivative with respect to time $t$ in the following form (Miller and Ross, 1993; Podlubny, 1999) 


$$
D_{t}^{\gamma} f(t) \equiv \frac{d^{\gamma}}{d t^{\gamma}} f(t) \equiv \frac{1}{\Gamma(1-\gamma)} \frac{d}{d t} \int_{0}^{t} \frac{f(\tau) d \tau}{(t-\tau)^{\gamma}}
$$

where $\Gamma(1-\gamma)$ is the Euler gamma function and $t \geqslant 0$ (Miller and Ross, 1993; Podlubny, 1999).

For many real materials, the fractional derivative order is commonly assumed to be in the interval $0<\gamma<1$ (Caputo and Mainardi, 1971a; Bagley and Torvik, 1983b; Enelund and Olsson, 1999). The constitutive relation with $\gamma=1$ represents Kelvin-Voight material with internal linear dissipation of mechanical energy (e.g. Mainardi, 2009; Hedrih (Stevanović), 2014)

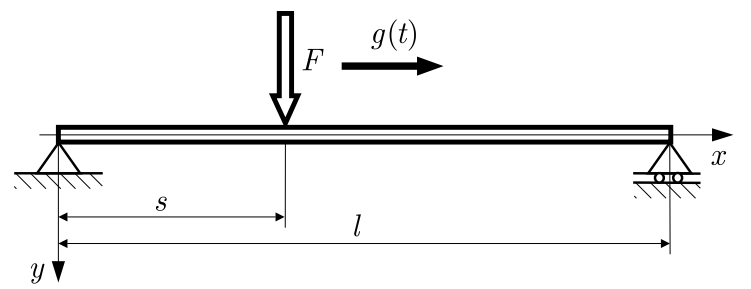

Fig. 1. Scheme of the analysed system

The beam is assumed to be subjected to a force moving with a constant velocity or acceleration from the left to the right edge of the beam, according with the sense of the $x$ axis (Fig. 1). Based on the above assumptions, the governing equation of the analysed beam is obtained as below

$$
E J\left(\frac{\partial^{4} y(x, t)}{\partial x^{4}}+\mu_{\gamma} \frac{d^{\gamma}}{d t^{\gamma}}\left(\frac{\partial^{4} y(x, t)}{\partial x^{4}}\right)\right)+A \rho \frac{\partial^{2} y(x, t)}{\partial t^{2}}=F \delta(x-g(t))
$$

where $A$ is the cross-section area of the beam, $J$ - axial moment of inertia of the beam cross-section respect to the neutral axis of the beam bending, $\rho$ - material mass density of the beam, $y(x, t)$ - transversal displacement of the neutral beam axis (Fig. 1), $t$ - time, $x$ - longitudinal coordinate, $F$-intensity of the external moving and concentrated force, $\delta$-Dirac delta function, $g(t)$ - function determining the force location, $g(t)=v t$ or $g(t)=\varepsilon t^{2} / 2$ for a force moving with a constant velocity or constant acceleration, respectively. Furthermore, this function must satisfy the following condition $0 \leqslant g(t) \leqslant l$.

For the simply-supported beam, the deflection and bending moment at both beam ends have to be zero, thus the boundary conditions are as below

$$
y(0, t)=0 \quad y(l, t)=0 \quad M_{b}(0, t)=0 \quad M_{b}(l, t)=0
$$

where $l$ is the length of the beam, $M_{b}$ - bending moment.

The bending moment is calculated as below

$$
M_{b}(x, t)=\int_{A} \sigma y d A=\int_{A}\left\{E \varepsilon(t)+E_{\gamma}^{\prime} D_{t}^{\gamma}[\varepsilon(t)]\right\} y d A=-E J\left\{\frac{d^{2} y(x, t)}{d x^{2}}+\mu_{\gamma} D_{t}^{\gamma}\left[\frac{d^{2} y(x, t)}{d x^{2}}\right]\right\}
$$

therefore

$$
\frac{d^{2} y(0, t)}{d x^{2}}=\frac{d^{2} y(l, t)}{d x^{2}}=0 \quad D_{t}^{\gamma}\left[\frac{d^{2} y(0, t)}{d x^{2}}\right]=D_{t}^{\gamma}\left[\frac{d^{2} y(l, t)}{d x^{2}}\right]=0
$$

Moreover, it is assumed that the beam is initially at rest and the initial bending moments are equal to 0. 
The solution to Eq. (2.3) is obtained utilizing the mode superposition principle (e.g. Kaliski, 1966; Clough and Penzien, 1993; Rao, 2004). The eigenfunctions for a simply supported beam are given by

$$
Y_{n}(x)=\sin \frac{n \pi x}{l} \quad n=1,2,3, \ldots
$$

Then, the forced-vibration solution of a beam can be expressed as

$$
y(x, t)=\sum_{n=1}^{\infty} \xi_{n}(t) Y_{n}(x)=\sum_{n=1}^{\infty} \xi_{n}(t) \sin \frac{n \pi x}{l}
$$

and the corresponding derivatives are evaluated below

$$
\frac{\partial^{4} y(x, t)}{\partial x^{4}}=\sum_{n=1}^{\infty} Y_{n}^{I V}(x) \xi_{n}(t) \quad \frac{d^{\gamma}}{d t}\left(\frac{\partial^{4} y(x, t)}{\partial x^{4}}\right)=\sum_{n=1}^{\infty} Y_{n}^{I V}(x) D_{t}^{\gamma}[\xi(t)]
$$

where

$$
D_{t}^{\gamma}\left[\xi_{n}(t)\right]=\frac{d^{\gamma} \xi(t)}{d t^{\gamma}}=\frac{1}{\Gamma(1-\gamma)} \frac{d}{d t} \int_{0}^{t} \frac{\xi_{n}(t) d \tau}{(t-\tau)^{\gamma}}
$$

and

$$
Y_{n}^{I V}(x)=\frac{d^{4} Y(x)}{d x^{4}} \quad \frac{\partial^{2} y(x, t)}{\partial t^{2}}=\sum_{n=1}^{\infty} Y_{n}(x) \ddot{\xi}_{n}(t)
$$

where

$$
\ddot{\xi}(t)=\frac{d^{2} \xi(t)}{d t^{2}}
$$

Substituting Eqs. (2.9) and (2.10) into (2.3), we get

$$
\sum_{n=1}^{\infty}\left(Y_{n}^{I V}(x) \xi_{n}(t)+\mu_{\gamma} Y_{n}^{I V}(x) D_{t}^{\gamma}\left[\xi_{n}(t)\right]+a_{b}^{2} Y(x) \ddot{\xi}_{n}(t)\right)=\frac{F}{E J} \delta(x-g(t))
$$

Next, integrating over $x$ from 0 to 1 , and using the orthogonality property of eigenfunctions, after some mathematical transformations, the following relationships are obtained

$$
\ddot{\xi}_{n}(t)+\mu_{\gamma} \omega_{n}^{2} D_{t}^{\gamma}\left[\xi_{n}(t)\right]+\omega_{n}^{2} \xi_{n}(t)=f_{0} \sin \frac{n \pi g(t)}{l}
$$

where

$$
f_{0}=\frac{2 F}{m} \quad \omega_{n}=\left(\frac{\pi n}{l}\right)^{2} \sqrt{\frac{E J}{\rho A}}
$$

$m$ - mass of the beam.

Assuming zero initial conditions

$$
\left.\xi(t)\right|_{t=0}=\left.0 \quad \frac{d[\xi(t)]}{d t}\right|_{t=0}=\left.0 \quad \frac{d^{\gamma-1}[\xi(t)]}{d t^{\gamma-1}}\right|_{t=0}=0
$$

the solution of equations (2.13) can be expressed as

$$
\xi_{n}(t)=f_{0} \int_{0}^{t} G_{n}(t-\tau) \sin \frac{n \pi g(\tau)}{l} d \tau
$$


where $G_{n}(t)$ is Green's function corresponding to Eq. (2.13) (Miller and Ross, 1993; Podlubmy, 1999; Rossikhin and Shitikova, 1997, 2010). This Green's function consists of two terms, namely

$$
G_{n}(t)=K_{1 n}(t)+K_{2 n}(t)
$$

The first term $K_{1 n}$ (Eq. (2.15)) represents damped vibrations around the drifting equilibrium position, while the second term $K_{2 n}$ describes the drift of the equilibrium position (Beyer and Kempfle, 1995; Rossikhin and Shitikova, 1997). The term $K_{1 n}$ could be calculated from the formula given by Beyer and Kempfle (1995)

$$
K_{1 n}(t)=\alpha_{n} \mathrm{e}^{-\sigma_{n} t} \sin \left(\Omega_{n} t+\phi_{n}\right)
$$

where

$$
\alpha_{n}=\frac{2}{\sqrt{\mu_{k}^{2}+\nu^{2}}} \quad \phi_{n}=\arctan \frac{\mu_{k}}{\nu} \quad \mu_{k}=\operatorname{Re}\left(W_{(p, 1,2)}^{\prime}\right) \quad \nu=\operatorname{Im}\left(W_{(p, 1,2)}^{\prime}\right)
$$

and $W(p)=p^{2}+\mu_{\gamma} \omega_{n}^{2} p^{\gamma}+\omega_{n}^{2}$ is the characteristic polynomial of equations (2.13), $W^{\prime}(p)=2 p+\gamma \mu_{\gamma} \omega_{n}^{2} p^{\gamma-1}$ - derivative of the characteristic polynomial with respect $p$, $p_{1,2}=-\sigma_{n} \pm \mathrm{i} \Omega_{n}$ - conjugate complex roots of the characteristic polynomial $W(p)$.

The term $K_{2 n}$ could be calculated using the formula (Beyer and Kempfle, 1995)

$$
K_{2 n}(t)=\frac{\mu_{\gamma} \omega_{n}^{2} \sin (\pi \gamma)}{\pi} \int_{0}^{\infty} \frac{r^{\gamma} \mathrm{e}^{-r t} d r}{\left[r^{2}+\mu_{\gamma} \omega_{n}^{2} r^{\gamma} \cos (\pi \gamma)+\omega_{n}^{2}\right]^{2}+\left[\mu_{\gamma} \omega_{n}^{2} r^{\gamma} \sin (\pi \gamma)\right]^{2}}
$$

In some cases of vibration analysis, $K_{2 n}$ could be neglected in comparison with $K_{1 n}$ (Kempfle et al., 2002; Rossikhin and Shitikova, 1997).

In the case of a viscoelastic integer order Kelvin-Voigt material model, the governing equation of the analysed beam has the following form (Kaliski, 1966)

$$
E J\left(\frac{\partial^{4} y(x, t)}{\partial x^{4}}+\mu \frac{\partial^{5} y(x, t)}{\partial x^{4} \partial t}\right)+A \rho \frac{\partial^{2} y(x, t)}{\partial t^{2}}=F \delta(x-g(t))
$$

The solution to the equation (2.18) could be obtained using a similar approach as in the case of the equation with fractional damping. For each $n$-th mode, the response could be calculated as follows (Kaliski, 1966)

$$
\xi_{n}(t)= \begin{cases}\frac{f_{0}}{\omega_{h n}} \int_{0}^{t} \mathrm{e}^{-h_{n}(t-\tau)} \sin \left(\omega_{h n}(t-\tau)\right) \sin \frac{n \pi g(\tau)}{l} d \tau & \text { for } \quad h_{n}<\omega_{n} \\ \frac{f_{0}}{\widetilde{\omega}_{h n}} \int_{0}^{t} \mathrm{e}^{-h_{n}(t-\tau)} \sinh \left(\widetilde{\omega}_{h n}(t-\tau)\right) \sin \frac{n \pi g(\tau)}{l} d \tau & \text { for } \quad h>\omega_{n}\end{cases}
$$

where

$$
h_{n}=\frac{1}{2} \mu \omega_{n}^{2} \quad \omega_{h n}=\sqrt{\omega_{n}^{2}-h_{n}^{2}} \quad \widetilde{\omega}_{h n}=\sqrt{h_{n}^{2}-\omega_{n}^{2}}
$$

It should be noted that when $\gamma=1$ (integer order derivative), the component $K_{2 n}$ of fractional Green's function (Eqs. (2.15) and (2.17)) vanishes, and it could be demonstrated that equations with a fractional and integer order derivative (Eq. (2.19)) are equivalent in the case of a subcritically damped system, i.e. $\omega_{n}>h_{n}$. 


\section{Calculation results and discussion}

In order to demonstrate the feasibility of the described above method, exemplary calculations of a beam subjected to a moving load have been performed. Responses of the beam with the fractional viscoelastic model are computed using equations (2.13)-(2.17) whereas the responses of the beam with the integer order derivative model are computed using equation (2.19).

The dimensionless dynamic deflection of the beam $y / y_{0}$ versus the dimensionless time parameter $\mathrm{s}$ has been computed. The variable $y_{0}$ denotes the static deflection at the beam mid-span and can be calculated from the equation

$$
y_{0}=\frac{F_{0} l^{3}}{48 E I}
$$

The dimensionless time parameter $\mathrm{s}$ is defined as

$$
s=\frac{v t}{l} \quad \text { or } \quad s=\frac{\varepsilon t^{2}}{2 l}
$$

in the case of a constant force velocity or constant acceleration, respectively.

The calculations are accomplished for several values of the order of fractional derivative $\gamma$, dimensionless velocity $v / v_{c r}$ and acceleration $\varepsilon / \varepsilon_{c r}$. Variables $v_{c r}$ and $\varepsilon_{c r}$ denote the critical velocity and acceleration, respectively. The critical velocity corresponds to the first natural vibration frequency of the beam and is defined as (Fryba, 1972)

$$
v_{c r}=\frac{\pi}{l} \sqrt{\frac{E J}{\rho A}}
$$

whereas the critical acceleration is defined as the acceleration at which the force at the end of the beam reaches the critical velocity and is defined as

$$
\varepsilon_{c r}=\frac{\pi^{2}}{l^{3}} \frac{E J}{\rho A}
$$

Firstly, the dynamic response of the beam subjected to a moving force at a constant velocity calculated with the help of presented above equations are compared to the results obtained using the corresponding formula given by Fryba (1972). These comparative calculations are performed assuming a very light damping i.e. $\mu_{\gamma}=1 \cdot 10^{-5} \mathrm{~s}^{\gamma}$ and $\gamma=1$ (integer order derivative). The calculations are performed for $v / v_{c r}=0.5$ (Eq. (3.1)). The calculation results obtained using the both equations are virtually identical. Minimal differences are found in the results, which is probably caused by differences in the modelling of damping in the system. Namely, in the equations given by Fryba (1972) it is assumed that the damping coefficient is independent of vibration modes and frequency in contrast to the damping model utilized in this work (Eqs. (2.3) and (2.13)). Next, in order to illustrate the application of the presented above procedure, computational examples for the response of the beam loaded with a moving force are performed. The calculations are carried out for the beam of length $20 \mathrm{~m}$, mass density $7600 \mathrm{~kg} / \mathrm{m}^{3}$, cross-section area $2 \cdot 10^{-3} \mathrm{~m}^{2}$, cross-section moment of inertia $3.953 \cdot 10^{-6} \mathrm{~m}^{4}$, Young's modulus $2.1 \cdot 10^{5} \mathrm{MPa}$, coefficient $\mu_{\gamma}=3 \cdot 10^{-2} \mathrm{~s}^{\gamma}$. The force value is assumed $F=100 \mathrm{~N}$.

From equation (2.16), it follows that the values of damping coefficients and vibration amplitudes depend on the roots of the characteristic equation and, therefore, knowledge of the roots is required for further calculations. The roots are computed for the varying fractional derivative order $\gamma$. The dependence of the roots of the characteristic equation on the fractional derivative order is shown in Fig. 2 (for the first two vibration modes). The real part of the root determines the damping coefficient whereas the imaginary part determines the vibration frequency (Eq. 
(a)

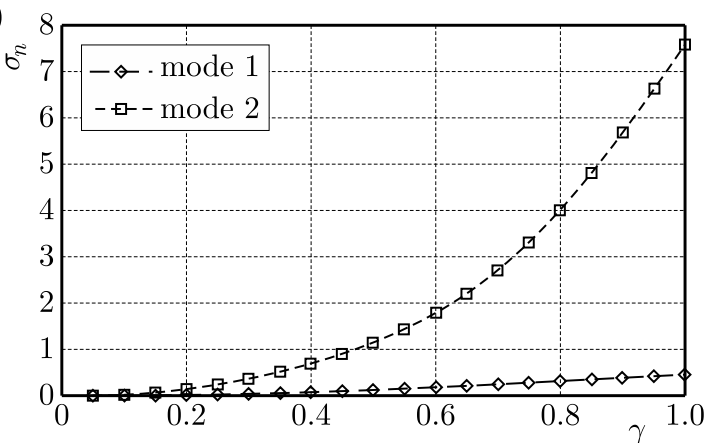

(b)

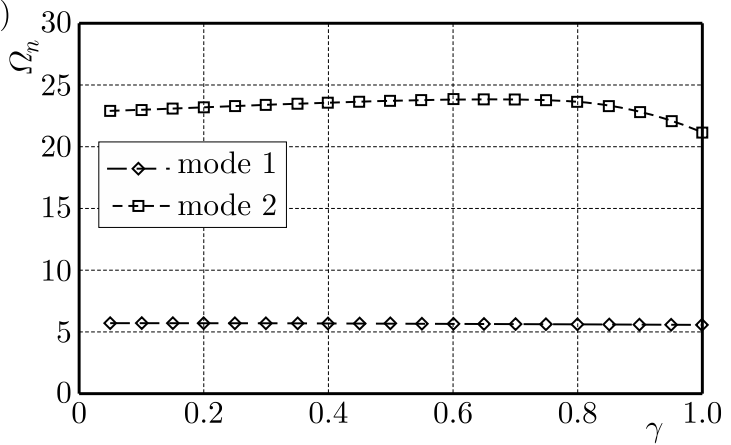

Fig. 2. Relationship between roots of the characteristic equation and the order of fractional derivative: (a) real part, (b) imaginary part

(2.16)). Performed calculations reveal that the value of the damping coefficient increases with the order of fractional derivative (Fig. 2a) but the vibration frequency is practically constant (Fig. 2b).

Moreover, the ratio of the real to the imaginary part of the roots of the characteristic equation increases with the increaasing order of fractional derivative (Fig. 3a), whereas the amplitude $\alpha_{n}$ (Eq. (2.16)) is practically independent of the order of fractional derivative (Fig. 3b). It could be expected that the dynamic deflection of the beam decreases with the increasing order of fractional derivative.

(a)

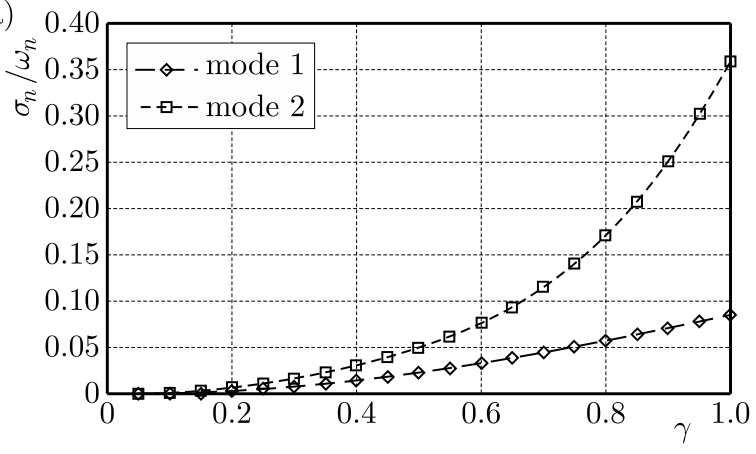

(b)

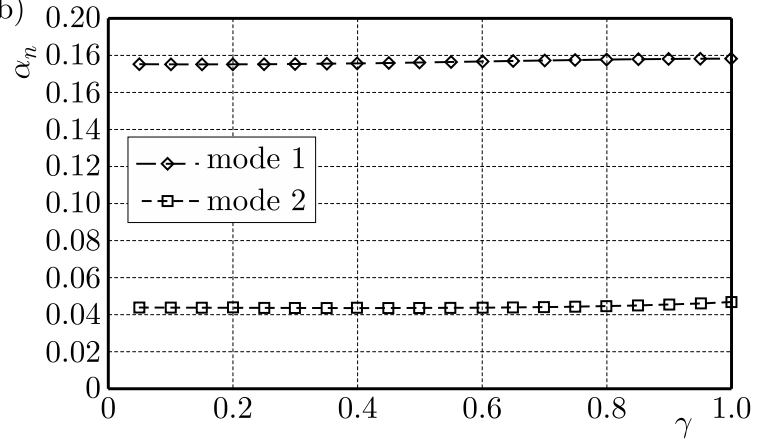

Fig. 3. (a) Ratio of the real to the imaginary part of the roots of the characteristic equation,

(b) relationship between the amplitude $\alpha_{n}$ and the order of fractional derivative

The responses of the beam subjected to a force moving at a constant velocity are presented in Figs. 4-6. In Fig. 4, the dimensionless dynamic deflection of the beam at the point under the travelling force at various values of the derivative order $\gamma$ is presented whereas the dynamic deflection at the mid-span of the beam is shown in Fig. 5. These results reveal that deflection of the beam under a moving force decreases with the increasing order of fractional derivative $\gamma$ (Fig. 4). The maximum deflection of the beam is greater for the dimensionless velocity $v / v_{c r}=0.5$ than $v / v_{c r}=1.0$. In the case $v / v_{c r}=0.5$, the dynamic deflection at the mid-span of the beam decreases with the increasing order of fractional derivative when $s<0.775$ while increases when $0.775<s<1.0$ (Fig. 5a). In the case $v / v_{c r}=1.0$, the dynamic deflection decreases with the increasing order of fractional derivative for the total duration of force action. In the case of a force moving at a constant velocity, for all computational examples, the maximum beam deflection is obtained when $\gamma=0.25$ (Figs. 5 and 6 )

A comparison between the dynamic deflections of the beam at various values of force velocity in the case of the derivative order $\gamma=0.5$ is shown in Fig. 6 . The response curves are similar to the corresponding curves computed for the integer order derivative presented in the literature (Fryba, 1972; Bajer and Dyniewicz, 2012; Abu Hilal and Zibdeh, 2000). 

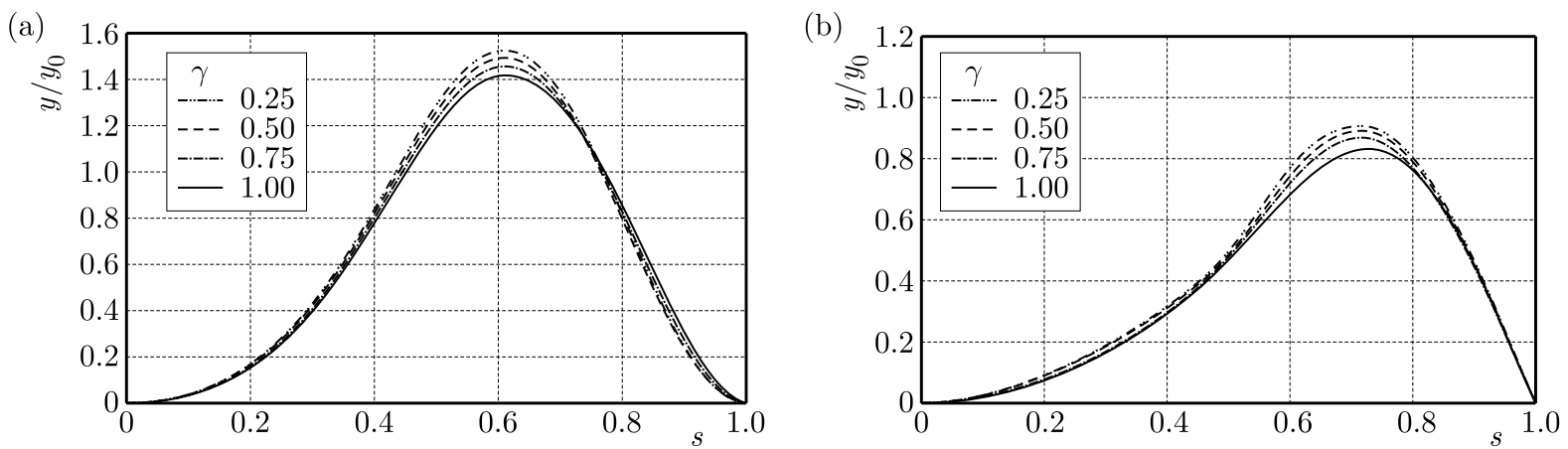

Fig. 4. Dynamic deflection of the beam under the moving force at various values of the derivative order $\gamma$, in the case of constant velocity: (a) $v / v_{c r}=0.5$, (b) $v / v_{c r}=1.0$

(a)

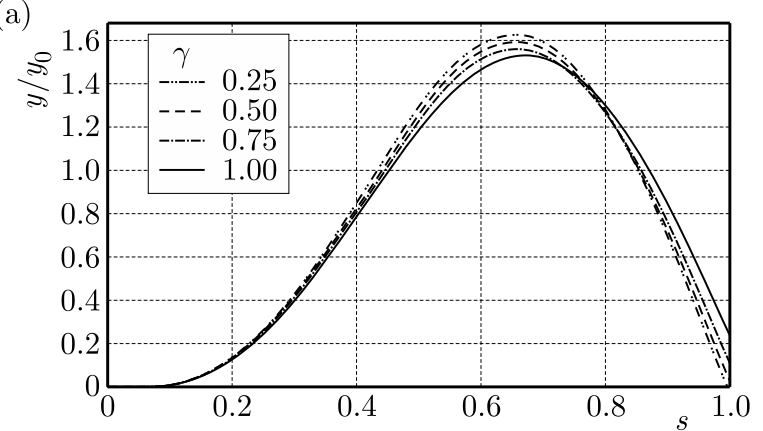

(b)

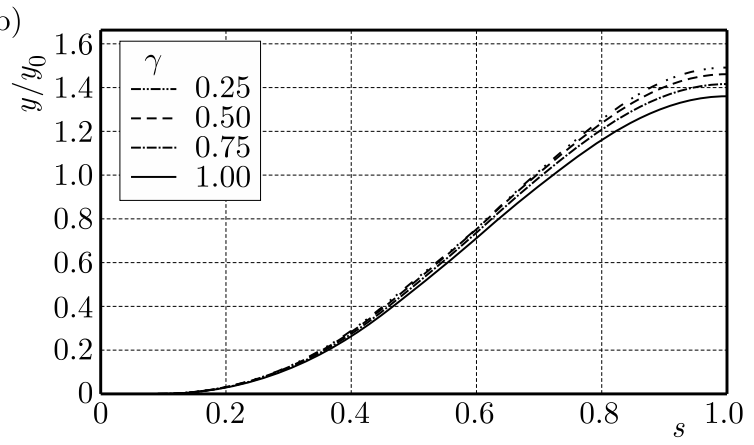

Fig. 5. Dynamic deflection at the mid-span of the beam at various values of the derivative order $\gamma$ in the case of constant velocity: (a) $v / v_{c r}=0.5$, (b) $v / v_{c r}=1.0$

(a)

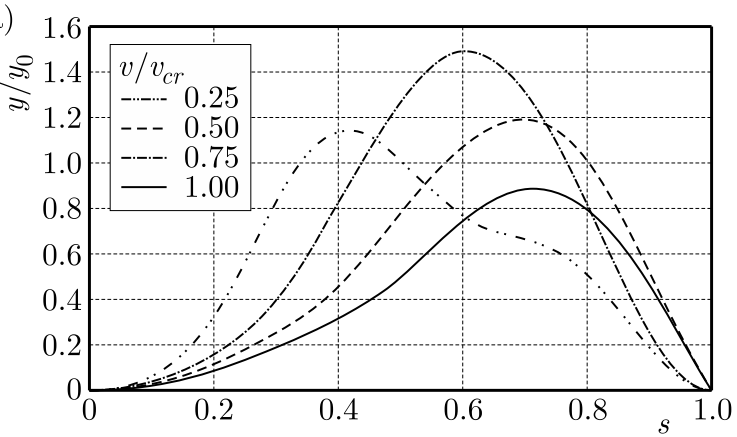

(b)

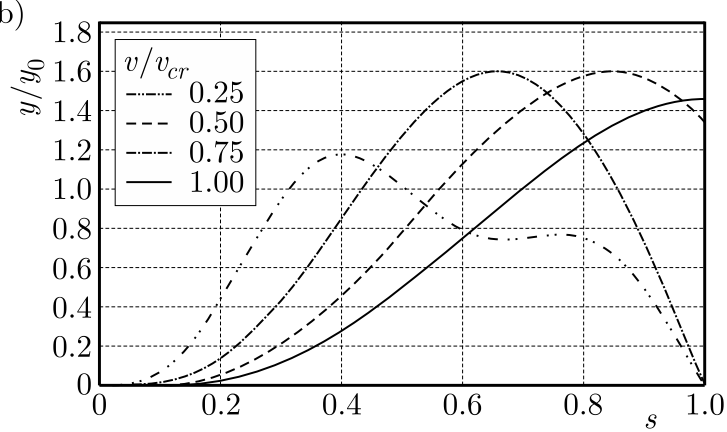

Fig. 6. A comparison between the dynamic deflection of the beam at various values of force velocity $v / v_{c r}$ in the case of the derivative order $\gamma=0.5$ : (a) under the travelling force, (b) at the mid-span of the beam

Beam responses to the force moving at a constant acceleration are presented in Figs. 7-9. In Fig. 7, the dimensionless dynamic deflection of the beam at the point under the travelling force at various values of the derivative order $\gamma$ is presented whereas the dynamic deflection at the mid-span of the beam is shown in Fig. 8. For all computational examples, the maximum beam deflection occurs in the case of $\gamma=0.25$ (Figs. 7 and 8). In the case of $\varepsilon / \varepsilon_{c r}=0.5$, the beam responses computed for various orders of the fractional derivatives differ slightly. The difference between curves obtained for $\varepsilon / \varepsilon_{c r}=0.5$ are smaller than for the curves obtained for $\varepsilon / \varepsilon_{c r}=1.0$ (Figs. 7 and 8).

In all calculation cases, the calculated values of the component $K_{2}$ of fractional Green's function are negligible in comparison to the values of the component $K_{1}$ (Eqs. (2.13)-(2.15)). Examples of $K_{1}$ and $K_{2}$ components of fractional Green's function as a function of the dimensionless time are shown in Fig. 10. 
(a)

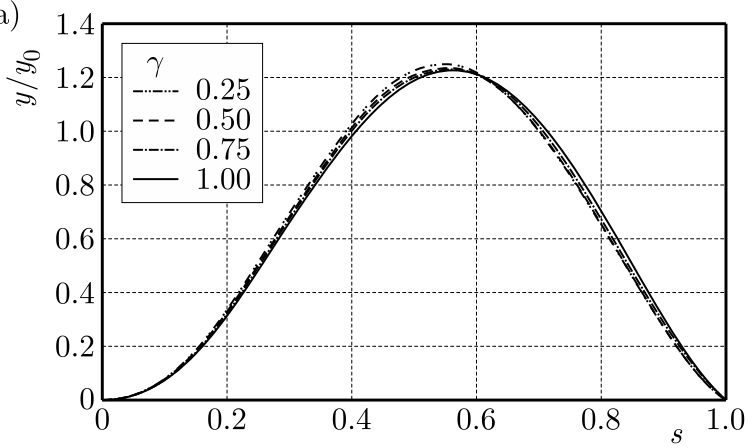

(b)

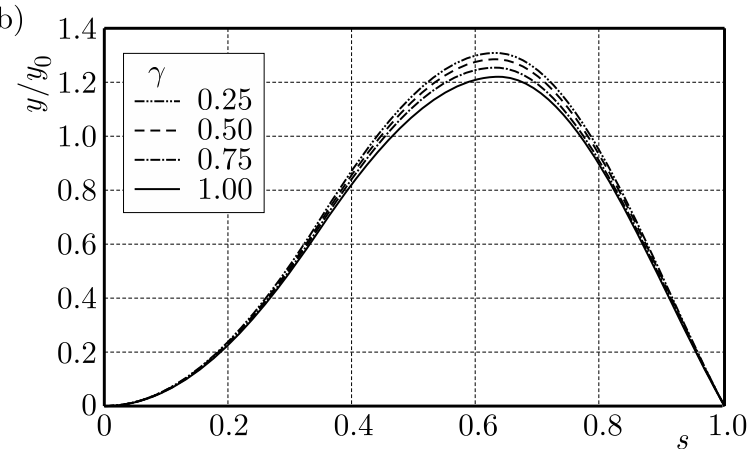

Fig. 7. Dynamic deflection of the beam under the travelling force at various values of the derivative order $\gamma$ in the case of dimensionless acceleration: (a) $\varepsilon / \varepsilon_{c r}=0.5$, (b) $\varepsilon / \varepsilon_{c r}=1.0$

(a)

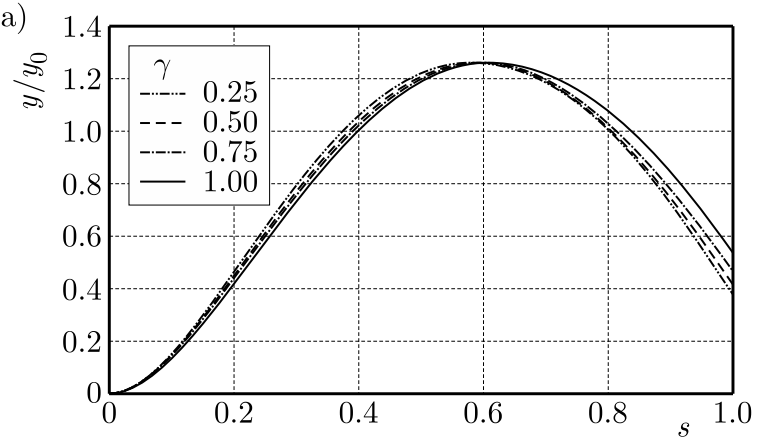

(b)

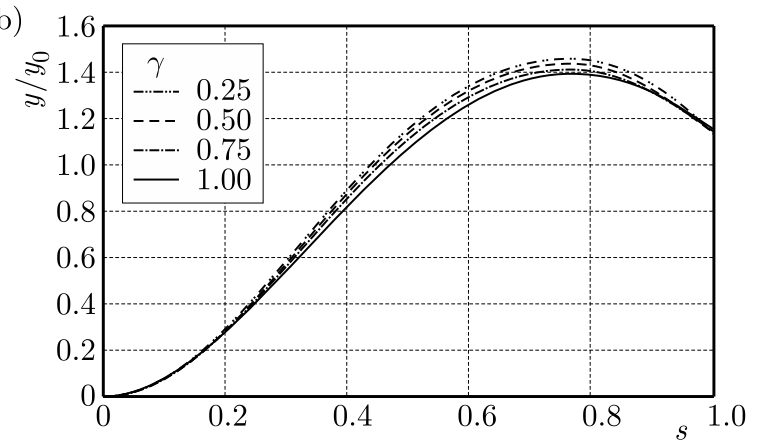

Fig. 8. Dynamic deflection at the mid-span of the beam at various values of the derivative order $\gamma$, in the case of dimensionless acceleration: (a) $\varepsilon / \varepsilon_{c r}=0.5$, (b) $\varepsilon / \varepsilon_{c r}=1.0$

(a)

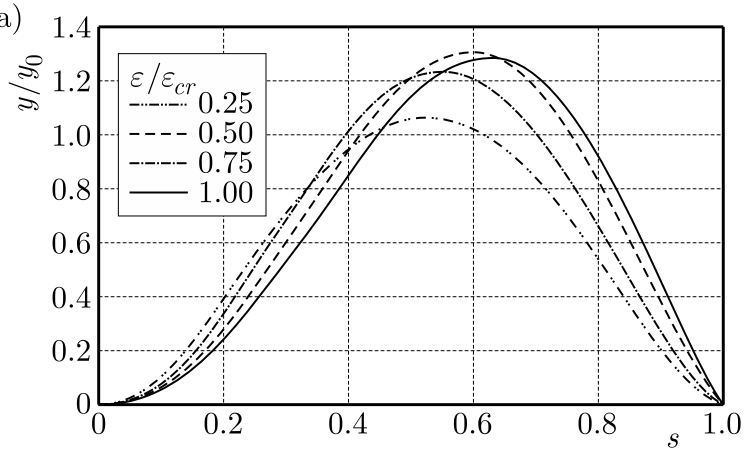

(b)

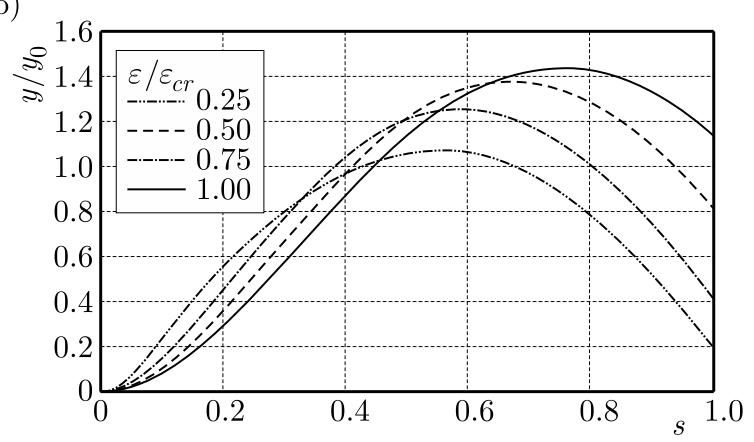

Fig. 9. A comparison between the dynamic deflection of the beam at various values of force acceleration $\varepsilon / \varepsilon_{c r}$ in the case of the derivative order $\gamma=0.5$ : (a) under the travelling force, (b) at the mid-span of the beam

(a)

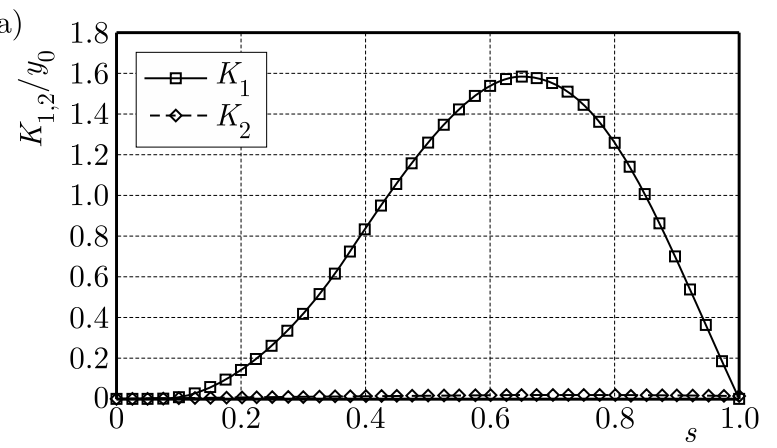

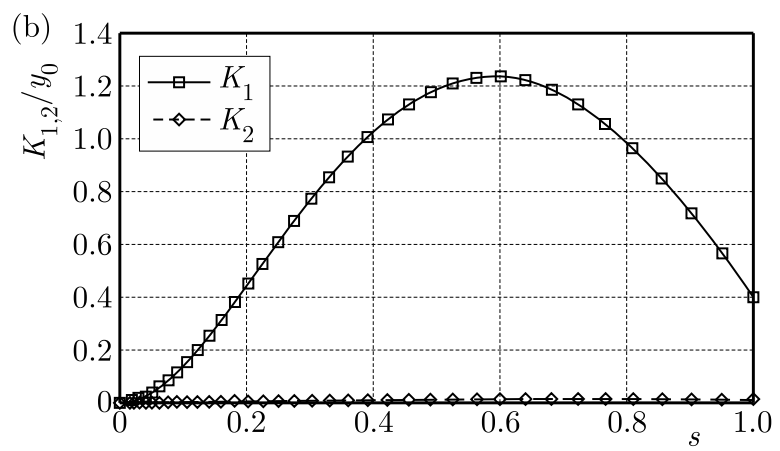

Fig. 10. Values of components $K_{1}$ and $K_{2}$ of fractional Green's function versus dimensionless time: (a) constant velocity, (b) constant acceleration 


\section{Conclusions}

In this paper, the governing equations of dynamic behaviour of a viscoelastic beam made of a material described by the fractional derivative Kelvin-Voigt model and subjected to a force travelling with a constant acceleration are presented. The solution to the governing equations is based on the method presented by Beyer and Kempfle (1995) or by Rossikhin and Shitikova (1997). The solution is achieved with the aid of a convolution integral of fractional Green's function and the forcing function. Green's function is formulated by two terms. The first term describes damped vibrations around the drifting equilibrium position, while the second term describes the drift of the equilibrium position. In the author's opinion, the proposed method of solution of fractional differential equations has advantages over the solution with Green's function in the form of the Mittag-Leffler series, which are presented in other works (Podlubny, 1996; Abu-Mallouh et al., 2012), because the mentioned series has a weak convergence rate. Furthermore, in some cases, the integral containing the second term of Green's function $K_{2}$ can be neglected (Eqs. (2.15) and (2.17)) (Rossikhin and Shitikova, 1997; Kempfle et al., 2012), which significantly reduces the time of numerical calculations.

Utilizing the obtained solution to the governing equations, exemplary calculations of a beam subjected to a moving load have been performed. In the first step, responses of the examined beam subjected to the travelling force with a constant velocity have been calculated using the obtained solution and formulae given by Fryba (1972). The calculation results obtained using both equations are virtually identical. Afterwards, responses of the beam subjected to a force moving at a constant and accelerated velocity have been computed. To the best of the author's knowledge, responses of a beam whose internal dissipation of mechanical energy is described by a differential equation of a fractional order and subjected to a force moving at a constant acceleration have not been published yet.

The performed calculations reveal that in the case of a force moving at a constant velocity, the calculated maximum deflection of the beam decreases with the increasing order of fractional derivative (Figs. 4 and 5) as could be expected (see section above). In the case of a force moving at a constant velocity, the calculated beam responses are similar to those presented in the works of other authors (e.g. Fryba, 1972; Bajer and Dyniewicz, 2012; Abu Hilal and Zibdeh, 2000).

In the case of a force moving at a constant acceleration, for all computational examples, the maximum beam deflection occurs when $\gamma=0.25$. In the case of $\varepsilon / \varepsilon_{c r}=0.5$, the beam responses computed for various orders of the fractional derivatives differ slightly. The difference between the responses computed for various orders of the fractional derivatives are smaller for $\varepsilon / \varepsilon_{c r}=0.5$ than for the responses obtained for $\varepsilon / \varepsilon_{c r}=1.0$.

The proposed approach expands the methods employing fractional calculus in the analysis of transient dynamic processes.

\section{A. Appendix}

Fractional Green's function of Eq. (2.13) can be found by the inverse Laplace transform of the expression below (Bagley and Torvik, 1983b; Beyer and Kempfle, 1995; Rossikhin and Shitikova, 1997; Podlubny, 1999)

$$
g(p)=\frac{1}{p^{2}+\mu_{\gamma} p^{\gamma} \omega_{0}^{2}+\omega_{0}^{2}}
$$

therefore

$$
G(t)=L^{-1}[g(p)]=\frac{1}{2 \pi \mathrm{i}} \int_{c-\mathrm{i} \infty}^{c+\mathrm{i} \infty} \frac{\mathrm{e}^{p t}}{p^{2}+\mu_{\gamma} p^{\gamma} \omega_{0}^{2}+\omega_{0}^{2}} d p
$$


The inverse transform integral is evaluated using Cauchy's residue theorem (Brown and Churchill, 2003). The function $g(p)$ has only two poles lying in the left complex plane (Beyer and Kempfle, 1995; Rossikhin and Shitikova, 1997). Moreover, the function $g(p)$ has a branch point at the origin, and the branch cut follows the negative real axis, then the negative real half-axis have to be encircled by the integration contour. The contour of integration, used in conjunction with the residue theorem, is shown in Fig. 11.

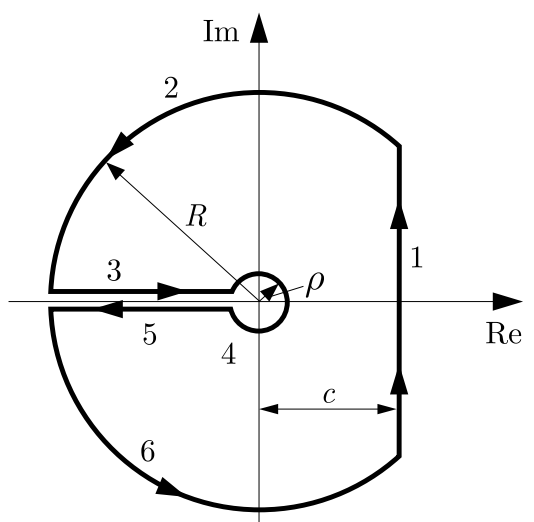

Fig. 11.

Therefore, it can be stated that

$$
\oint_{L_{123456}} G(p) d p=2 \pi \mathrm{i} \sum_{k=1}^{2} r e z_{p_{k}} g(p)
$$

and

$$
\begin{aligned}
& \oint_{L_{1}} g(p) d p+\oint_{L_{2}} g(p) d p+\oint_{L_{2}} g(p) d p+\oint_{L_{4}} g(p) d p+\oint_{L_{5}} g(p) d p+\oint_{L_{6}} g(p) d p \\
& \quad=2 \pi \mathrm{i} \sum_{k=1}^{n} r e z_{p_{k}} g(p)
\end{aligned}
$$

but

$$
G(t)=\frac{1}{2 \pi \mathrm{i}} \oint_{L_{1}} g(p) d p
$$

thus

$$
\begin{aligned}
& \oint_{L_{1}} g(p) d p=-\left(\oint_{L_{2}} g(p) d s+\oint_{L_{3}} g(p) d p+\oint_{L_{4}} g(p) d p+\oint_{L_{5}} g(p) d p+\oint_{L_{6}} g(p) d p\right) \\
& \quad+2 \pi \mathrm{i} \sum_{k=1}^{n} r e z_{p_{k}} g(p)
\end{aligned}
$$

It could be shown that the integrals along contours $L_{2}, L_{4}, L_{6}$ are equal to 0 . The integrals along the contours $L_{3}$ and $L_{5}$ could be evaluated using substitution $p=r \mathrm{e}^{\mathrm{i} \pi}$ and $p=r \mathrm{e}^{-\mathrm{i} \pi}$. Noting 
that $r \mathrm{e}^{\mathrm{i} \pi}=r \mathrm{e}^{-\mathrm{i} \pi}=r(\cos \pi \pm \mathrm{i} \sin \pi)=-r, p=-r$ and $d p=-d r$, the integrals $I_{3}$ and $I_{5}$ along the contours $L_{3}$ and $L_{5}$ can be evaluated as below

$$
\begin{aligned}
I_{3} & +I_{5}=\int_{L_{3}} \frac{\mathrm{e}^{p t}}{p^{2}+\mu_{\gamma} \omega_{0}^{2} p^{\gamma}+\omega_{0}^{2}} d p+\int_{L_{5}} \frac{\mathrm{e}^{p t}}{p^{2}+\mu_{\gamma} \omega_{0}^{2} p^{\gamma}+\omega_{0}^{2}} d p \\
& =\int_{R}^{0} \frac{-\mathrm{e}^{-r t}}{r^{2} \mathrm{e}^{\mathrm{i} 2 \pi}+\mu_{\gamma} \omega_{0}^{2} r^{\gamma} \mathrm{e}^{\mathrm{i} \gamma \pi}+\omega_{0}^{2}} d r+\int_{0}^{R} \frac{-\mathrm{e}^{-r t}}{r^{2} \mathrm{e}^{-\mathrm{i} 2 \pi}+\mu_{\gamma} \omega_{0}^{2} r^{\gamma} \mathrm{e}^{-\mathrm{i} \gamma \pi}+\omega_{0}^{2}} d r
\end{aligned}
$$

and

$$
\begin{aligned}
I_{3}+ & I_{5}=\int_{R}^{0} \frac{-\mathrm{e}^{-r t}}{r^{2}+\mu_{\gamma} \omega_{0}^{2} r^{\gamma}[\cos (\pi \gamma)+\mathrm{i} \sin (\pi \gamma)]+\omega_{0}^{2}} d r \\
& +\int_{0}^{R} \frac{-\mathrm{e}^{-r t}}{r^{2}+\mu_{\gamma} \omega_{0}^{2} r^{\gamma}[\cos (\pi \gamma)-\mathrm{i} \sin (\pi \gamma)]+\omega_{0}^{2}} d r=\int_{R}^{0} \frac{-\mathrm{e}^{-r t}(\mathcal{A}-\mathrm{i} \mathcal{B})}{\mathcal{A}^{2}+\mathcal{B}^{2}} d r \\
& +\int_{0}^{R} \frac{-\mathrm{e}^{-r t}(\mathcal{A}+\mathrm{i} \mathcal{B})}{\mathcal{A}^{2}+\mathcal{B}^{2}} d r=\int_{0}^{R} \frac{\mathrm{e}^{-r t}(\mathcal{A}-\mathrm{i} \mathcal{B}}{\mathcal{A}^{2}+\mathcal{B}^{2}} d r+\int_{0}^{R} \frac{-\mathrm{e}^{-r t}(\mathcal{A}+\mathrm{i} \mathcal{B})}{\mathcal{A}^{2}+\mathcal{B}^{2}} d r \\
& =\int_{0}^{R} \frac{-2 \mu_{\gamma} \omega_{0}^{2} r^{\gamma} \mathrm{e}^{-r t} \mathrm{i} \sin (\pi \gamma)}{\mathcal{A}^{2}+\mathcal{B}^{2}} d r
\end{aligned}
$$

where

$$
\begin{aligned}
& \mathcal{A}=r^{2}+\mu_{\gamma} \omega_{0}^{2} r^{\gamma} \cos (\pi \gamma)+\omega_{0}^{2} \\
& \mathcal{B}=\mu_{\gamma} \omega_{0}^{2} r^{\gamma} \sin (\pi \gamma)
\end{aligned}
$$

Denoting the sum of integrals in the RHS of Eq. (A.8) as $K_{2}$, and for $R \rightarrow \infty$, the following relationship is obtained

$$
K_{2}(t)=\frac{-1}{2 \pi \mathrm{i}} \int_{0}^{\infty} \frac{-2 \mu_{\gamma} \omega_{0}^{2} r^{\gamma} \mathrm{e}^{-r t} \mathrm{i} \sin (\pi \gamma)}{\mathcal{A}^{2}+\mathcal{B}^{2}} d r=\frac{\mu_{\gamma} \omega_{0}^{2} \sin (\pi \gamma)}{\pi} \int_{0}^{\infty} \frac{r^{\gamma} \mathrm{e}^{-r t}}{\mathcal{A}^{2}+\mathcal{B}^{2}} d r
$$

\section{References}

1. Abu Hilal M., Zibdeh H.S., 2000, Vibration analysis of beams with general boundary conditions traversed by a moving force, Journal of Sound and Vibration, 229, 2, 377-388

2. Abu-Mallouh R., Abu-Alshaikh I., Zibdeh H.S, Ramadan. K., 2012, Response of fractionally damped beams with general boundary conditions subjected to moving loads, Shock and Vibration, 19, 333-347

3. Alkhaldi H.S., Abu-Alshaikh I.M., Al-Rabadi A.N., 2013, Vibration control of fractionallydamped beam subjected to a moving vehicle and attached to fractionally-damped multi-absorbers, Advances in Mathematical Physics, ID 232160, http://dx.doi.org/10.1155/2013/232160

4. Atanackovic T.M., Stankovic B., 2002, Dynamics of a viscoelastic rod of fractional derivative type, $Z A M M, \mathbf{8 2}, 6,377-386$

5. Bagley R.L., Torvik P.J., 1983a, A theoretical basis for the application of fractional calculus to viscoelasticity, Journal of Rheology, 27, 3, 201-210 
6. Bagley R.L., Torvik P.J., 1983b, Fractional calculus - a different approach to the analysis of viscoelastically damped structures, AIAA Journal, 21, 5, 741-748

7. Bagley R.L., Torvik P.J., 1986, On the fractional calculus model of viscoelastic behavior, Journal of Rheology, 30, 1, 133-155

8. Bajer C.I., Dyniewicz B., 2012, Numerical Analysis of Vibrations of Structures under Moving Inertial Load, Springer, Heidelberg

9. Beyer H., Kempfle S., 1995, Definition of physically consistent damping laws with fractional derivatives $Z A M M, 75,8,623-635$

10. Brown J.W., Churchill R.V., 2003, Complex Variables and Applications, 7th ed., McGraw-Hill, Boston

11. CAputo M., 1974, Vibrations of an infinitive viscoelastic layer with a dissipative memory, Journal of Acoustical Society of America, 56, 3, 897-904

12. Caputo M., Mainardi F., 1971a, A new dissipation model based on memory mechanism, Pure and Applied Geophysics, 91, 8, 134-147

13. Caputo M., Mainardi F., 1971b, Linear models of dissipation in anelastic solids, Rivista del Nuovo Cimento, 1, 2, 161-198

14. Clough R.W., Penzien J., 1993, Dynamics of Structures, MacGraw-Hill, New York

15. Enelund M., Olsson P., 1999, Damping described by fading memory analysis and application to fractional derivative models, International Journal of Solids and Structures, 36, 939-970

16. FrYBA L., 1972, Vibration of Solids and Structures under Moving Loads, Noordhoff International Publishing, Groningen, The Netherlands

17. Hedrih (Stevanović) K.R., 2014, Generalized functions of fractional order dissipation of energy system and extended lagrange differential equations in matrix form, Tensor, 75, 1, 35-51

18. Hedrih (Stevanović) K.R., Filipovski A., 2002, Longitudinal creep vibrations of a fractional derivative order rheological rod with variable cross section, Facta Universitatis, Series Mechanics, Automatic Control and Robotics, 3, 12, 327-349

19. Hedrih (Stevanović) K.R., Machado T.J.A., 2015, Discrete fractional order system vibrations, International Journal of Non-Linear Mechanics, 73, 2-11

20. Kaliski S. (ED.), 1966, Vibrations and Waves in Solids (in Polish), Państwowe Wydawnictwo Naukowe, Warszawa

21. Kempfle S, Schäfer I., Beyer H., 2002, Fractional calculus via functional calculus: theory and application, Nonlinear Dynamics, 29, 99-127

22. MAinARDi F., 2009, Fractional Calculus and Waves in Linear Viscoelastisity: an Introduction to Mathematical Models, Imperial College Press, London

23. Miller K.S., Ross B., 1993, An Introduction to the Fractional Calculus and Fractional Differential Equations, John Willey \& Sons Inc., New York

24. Podlubny I., 1999, Fractional Differential Equations, Academic Press, San Diego

25. Rao S., 2004, Mechanical Vibrations, 4-th Ed., Prentice Hall, Upper Saddle River

26. Rossikhin Y.A., Shitikova M.V., 1997, Application of fractional derivatives to the analysis of damped vibrations of viscoelastic single mass systems, Acta Mechanica, 120, 109-125

27. Rossikhin Y.A., Shitikova M.V., 2010, Application of fractional calculus for dynamic problems of solid mechanics: novel trends and recent results, Applied Mechanics Reviews, 63, 1-51 